\title{
Modifications to automation project of a Hyundai genset in Cayo Santa Maria
}

\author{
Julian Javier Valdivia de Armas ${ }^{1}$, Emilio Jiménez Leyva ${ }^{2}$ \\ ${ }^{1,2}$ Universidad Central "Martha Abreu” de las Villas (UCLV) Carretera a Camajuaní Km. 5 y 1/2. Santa Clara, Cuba \\ Email: javier@ativc.une.cu
}

Received: November $24^{\text {th }}, 2017$.

ABSTRACT

Accepted: November $30^{\text {th }}, 2017$.

Published: December $13^{\text {th }}, 2017$.

The paper presents the integration of one battery of Hyundai Engines 1.7 MW to the isolated system Cayo Santa Maria. Including the adaptation of the automation project, preparation of drawings and panels. Preparation of SCADA, PLC programming and configuration of the communication.

Copyright $\odot 2016$ by authors and Institute of Technology Galileo of Amazon (ITEGAM). This work is licensed under the Creative Commons Attribution International License (CC BY 4.0).

http://creativecommons.org/licenses/by/4.0/

Keywords: Programmable Controllers, Programming, SCADA systems.

\section{Modificaciones al proyecto de automática del grupo electrógeno Hyundai en el Cayo Santa María}

\section{RESUMEN}

El documento presenta la integración de una batería de Hyundai Engines 1.7 MW para el sistema aislado Cayo Santa María. Incluyendo la adaptación del automatización de proyectos, preparación de los diseños y los paneles. Preparación de SCADA, PLC programación y configuración de la comunicación.

Palabras clave: Controladores programables, programación, sistemas SCADA.

\section{INTRODUCTION}

Como parte del desarrollo económico de Cuba se comienza la explotación de los recursos naturales propios, en función de la industria turística. Por las grandes distancias que separan la Cayería Norte de la provincia de Villa Clara de la costa fue necesario emplazar una UEB de Generación, que con el crecimiento de las instalaciones permitiera ampliar la potencia instalada hasta ese momento.

A razón del vertiginoso crecimiento turístico de Cayo SantaMaria en el año 2013 se decidió acoplar a ese sistema aislado una Batería de motores Hyundai de 1,7 MW, para incrementar la potencia instalada en aproximadamente $7 \mathrm{MW}$.

Los sistemas Hyundai vienen en forma de paquetes de 8 , 12 y 16 motores, denominados baterías como los que se encuentran distribuidos por todo el país.

Para llevar a cabo la ejecución de esta adaptación se realiza una evaluación de qué sistemas del proyecto original debían mantenerse y cuáles no eran vitales para el funcionamiento de la misma. Además se debían hacer los acoples con la tecnologia MAN existente.

El objetivo general del proyecto es: acoplar una batería de motores Hyundai 1,7 MW al sistema aislado de Cayo Santa María y como específicos: modificar la programación de los autómatas de HTU y RCMS para la nueva configuración y rediseñar el SCADA del fabricante.

\section{TAREAS DEL PROYECTO}

Con el inicio de la Revolución Energética impulsada por el Comandante en Jefe Fidel Castro Ruz a partir del 1ro de enero de 2006, se logró llevar la producción de la energía eléctrica más cerca del punto final de consumo, ya que en nuestro país toda la generación eléctrica estaba basada hasta ese entonces em el uso de Centrales Termoeléctricas con las consecuentes pérdidas por transmisión en las líneas.

\section{II.1 DESCRIPCIÓN DEL PROCESO}

Una central eléctrica con motores, es un proceso donde se genera electricidad a partir del movimiento de un motor de combustión interna al que se acopla un generador eléctrico. Este tipo de central es usada de dos formas a régimen continuo (base) sincronizadas al SEN (sistema electro energético nacional) o en isla (cuando operan separadas). Para su uso en el Cayo complementan la generación como sistema aislado, siendo usados básicamente en modo base sincronizadas a los generadores MAN. 
Una central eléctrica con motores fuel generalmente consta de las siguientes unidades:

- Compresor: genera el aire de arranque del motor diesel y accionamiento de la instrumentación.

- Tratamiento de agua: produce el agua tratada para el enfriamiento del motor, generación de vapor en caldera y em purificadoras de aceite y combustible.

- Generador de vapor: produce el vapor para el tratamento del combustible.

- Preparación de combustible y lubricante: elimina sólidos, agua en el combustible y restos de la combustión en el aceite.

- Motor generador: produce la electricidad, equipo principal de la central.

Cada una de las unidades mencionadas anteriormente posee su propio automatismo y a la vez se enlazan entre sí en un único sistema, el SCADA donde se supervisa la central como um conjunto y con ello el proceso de generación de electricidad.

Cada unidad posee un PLC (controlador lógico programable), que ejecuta una rutina específica para cada una, además recolecta y envía eventos, estados, disparos y datos a través de una red de Ethernet Industrial hasta las PC, donde se procesa, salva y visualiza la información por el SCADA. [12]

Al trasladar la Central Eléctrica de Naranjito por problemas en la ubicación en la Ciudad de la Habana, se desmontó esta planta de 16 motores. Esto brindó la posibilidad de usar 4 motores para ampliar la capacidad de generación en el Cayo Santa María. Por lo que se decidió a trasladar una batería

completa.

Las baterías de Hyundai están conformadas por 4 motores (MDU), 1 caldera recuperadora, 2 compresores y 1 unidad de tratamiento de combustible (HTU). Cada central cuenta además con una Planta de Tratamiento Químico de Agua (PTQA), uma Estación de Bombeo y un cuarto de Control Remoto y Monitoreo (RCMS) en el cual se ubica el SCADA de la planta. Se decidió trasladar solamente el RCMS diseñado para 16 motores, la batería de motores y las bombas de transferencia sin su panel de control.

Con esto fue necesario acometer las siguientes tareas:

- Integración de bombas de transferencia al HTU.

- Adaptación de la configuración del RCMS para 4 motores.

- Adecuación del SCADA para operar 1 batería.

\section{IMPLEMENTACIÓN DEL PROYECTO}

Para la integración a la planta de la Batería Hyundai, prévio análisis se decidió que no era fundamental trasladar todos los módulos que conformaban el sistema.

\section{III.1 INTEGRAR BOMBAS DE TRANSFERENCIA AL HTU}

Las bombas de transferencia de combustible, forman parte de la Estación de Bombeo la cual no fue trasladada. Dicha estación pertenece a los módulos que conforman la planta agrupados em bombas de descarga y transferencia. Las bombas de descarga son las que llevan el combustible tanto el diesel como el fuel-oíl a los tanques de almacenamiento. Cuya parte del proceso ya existía y no era necesario su uso en el proyecto. Las bombas de transferencia son las encargadas de llevar el combustible desde los tanque de almacenamiento a los tanques de servicio del HTU, por lo que estas si eran parte indispensable para realizar la integración, debido a que no había compatibilidad entre el sistema de MAN y el de Hyundai.

En los proyectos originales de Hyundai es el HTU el que gobierna el arranque y parada de las bombas de transferencia, comandos programados y previamente enviados por comunicación hacia el PLC de la Estación de Bombeo. Al activarse las señales de bajo nivel de los tanques de servicio de diesel ó fuel-oíl, se enviaba la señal de arranque de las respectivas bombas de transferencias al PLC de la estación de bombeo. Como este panel no fue necesario trasladarlo se debía analizar el programa del PLC del HTU y realizar un diagnóstico de las entradas y salidas disponibles para ejecutar esta función ahora de forma local. Además de poder señalizar en el panel el arranque y parada de las bombas y realizar la selección de operación de las bombas en caso de que una estuviera en fala así como su trabajo automático.

Quedando conformado de la siguiente manera en el anunciador mostrando señal de alarma en las bombas de transferencia de HFO y Diesel (DO). En las lámparas de indicación se muestra cual bomba de transferencia se encuentra en operación.

\begin{tabular}{|c|c|c|c|c|c|}
\hline \multicolumn{5}{|c|}{ FI (ANNUNCIATOR) DETAIL } & \\
\hline \multicolumn{5}{|c|}{ DC24V LED LAMP (RED COLOR) } & \\
\hline $\begin{array}{c}\text { F.O SERVICE } \\
\text { TANK LEVE } \\
\text { LOW }\end{array}$ & $\begin{array}{l}\text { D.O SERVICE } \\
\text { TANK LEVEL } \\
\text { LOW }\end{array}$ & $\begin{array}{l}\text { D.O SERVICE } \\
\text { TANK IEVEL } \\
\text { HIGH }\end{array}$ & $\begin{array}{l}\text { H.F.O LEVEL } \\
\text { CONTROL V/N } \\
\text { ALARM }\end{array}$ & $\begin{array}{l}\text { H.F.O LEVEL } \\
\text { CONTROL V/N } \\
\text { ALARM }\end{array}$ & \\
\hline $\begin{array}{l}\text { F.0 } \\
\text { BOOSTER P/P } \\
\text { PRESS. } \\
\text { LOW }\end{array}$ & $\begin{array}{c}\text { F.0 FILTER } \\
\text { DIFE. } \\
\text { PRES. }\end{array}$ & 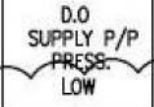 & & & \\
\hline $\begin{array}{l}\text { BOILAR } \\
\text { COMMON } \\
\text { ALARM }\end{array}$ & $\begin{array}{c}\text { ALARMA BONBA } \\
\text { TRANSFERENCIA } \\
\text { H.F.O. } 1\end{array}$ & $\begin{array}{c}\text { ALARNA BONBA } \\
\text { TRANSFERENCIA } \\
\text { H.F.O. } \# 2\end{array}$ & $\begin{array}{c}\text { ALARMA BONBA } \\
\text { TRANSFERENCIA } \\
\text { D.0. } \# 1\end{array}$ & $\begin{array}{c}\text { ALARMA BOMBA } \\
\text { TRANSFERENCIA } \\
\text { D.0. } 2\end{array}$ & $\Delta$ \\
\hline
\end{tabular}

Figura 1: Nuevas variables incorporadas al anunciador de panel de HTU.

Fuente: Autores, (2017).

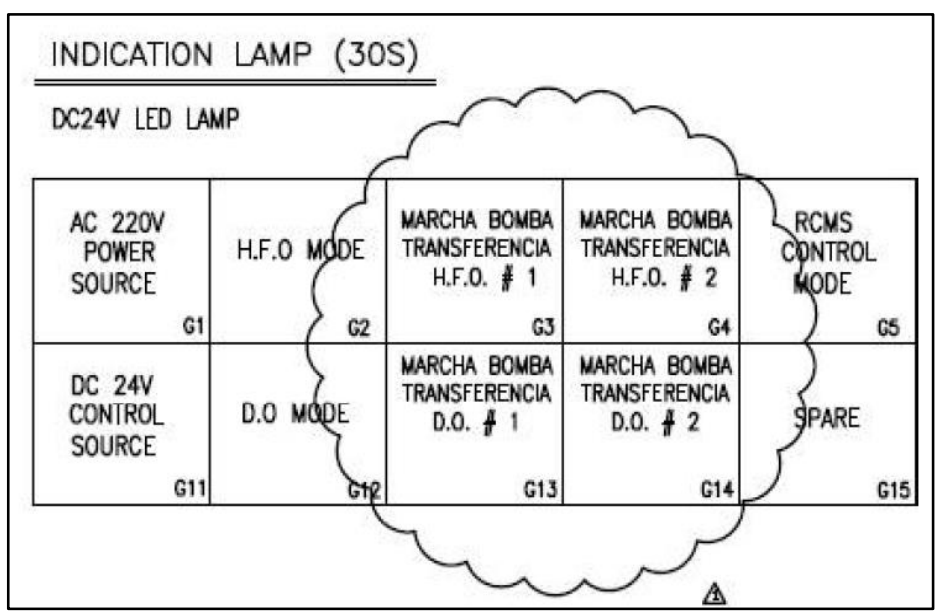

Figura 2: Nuevas variables incorporadas indicador del panel de HTU.

Fuente: Autores, (2017).

Como parte de la modificación a la lógica del PLC del HTU se utilizaron entradas digitales que estaban como repuesto en el autómata, para las señales de Marcha Bomba Transferencia HFO\#1 y \#2 se utilizaron las entradas I4.0 e 
respectivamente. Para las señales de Marcha Bomba Transferencia DO \#1 y \#2 se usaron las entradas I4.3 e I4.4, tal como se muestra en la figura3.

\begin{tabular}{|c|c|c|c|c|c|}
\hline \multicolumn{2}{|c|}{$\begin{array}{l}\text { EEST } \\
321-18 L 00-0 A A O\end{array}$} & \begin{tabular}{ll|l|} 
LOCAL RACK NO. & 0 & SLOT NO. \\
\end{tabular} & \multicolumn{2}{|c|}{ REEEREICE } & \\
\hline TB NO. & ADO NO. & DESCRPIION & FROM & LOCATON & \\
\hline $\mathrm{A} / 2$ & $1: 4.0$ & WARCHA BOMBA TRANSFERENCIA H.F.O. 1 & & $\begin{array}{l}\text { P. CONTROL } \\
\text { BONBSS COMB }\end{array}$ & $\Delta$ \\
\hline $\mathrm{A} / 3$ & $1: 4.1$ & USCOSTYY CONMON ALABM & & $\begin{array}{l}\text { Frou } \\
\text { UScostry }\end{array}$ & \\
\hline$\sqrt{4}$ & $Y: 4.2$ & MARCHA BOMBA TRANSFERENCIA H.F.O. \#2 & & & \\
\hline N/5 & $1: 4.3$ & WARCHA BOMBA TRANSFERENCIA D.0. 1 & & P. CONTROL & $\Delta$ \\
\hline A/6 & $1: 4.4$ & IARCHA BOMBA TRANSFERENCIA D.0. 2 & & & \\
\hline$\sqrt{7}$ & h.5.5 & ALARUA BOMBA TRANSFERENCIA H.F.O. I 1 & & & \\
\hline
\end{tabular}

Figura 3: Entradas utilizadas para señales de las bombas de transferencia.

Fuente: Autores, (2017).

Fue necesario además incorporar un Switch en el panel del HTU para poder cumplir con los requisitos de distancia de la red Ethernet Industrial.

\section{2 ADAPTAR LA CONFIGURACIÓN DEL RCMS PARA 4 MOTORES}

Fue necesario reajustar todo el hardware del panel del RCMS para el control de solo 4 motores, puesto que el panel original controlaba y monitoreaba la operación de 16 motores iban a quedar módulos de entradas y salidas, así como de comunicación de forma obsoleta. De igual forma se tuvo que modificar todo el programa del autómata del control para que fuera consistente el trabajo de hardware y software.

La configuración original tal y como se puede apreciar en la figura 4 consta de:

- 1 módulo de expansión IM365 (Interface para um bastidor de ampliación).

- módulos de comunicación CP340 (Procesador de comunicación con conexión RS422/485).

- 2 módulos de 32 entradas digitales (Módulo de entradas digitales DI32 24V).

- 7 módulos de 32 salidas digitales (Módulo de salidas digitales DO32 24V/0.5A, en grupos de 8).

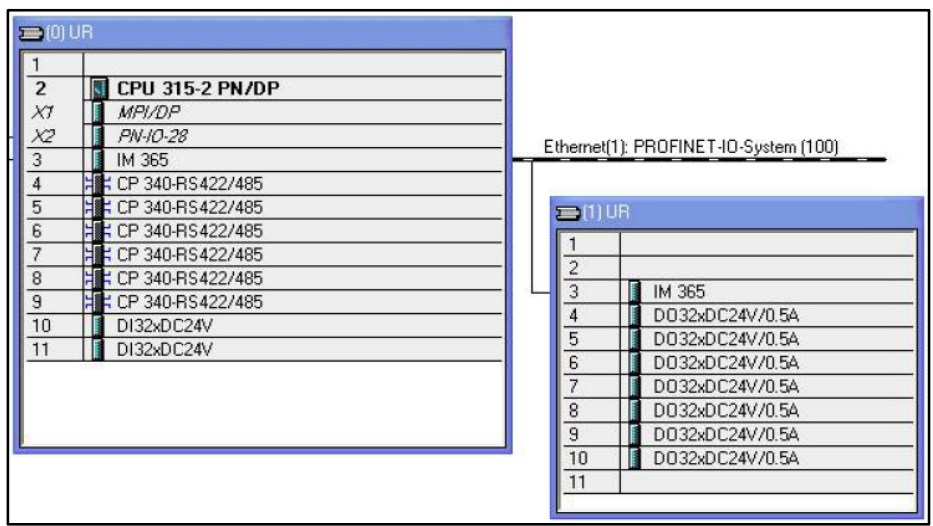

Figura 4: Configuración de Hardware original del PLC de RCMS para 16 motores (Vista desde el HW Configuración de STEP7). Fuente: Autores, (2017).

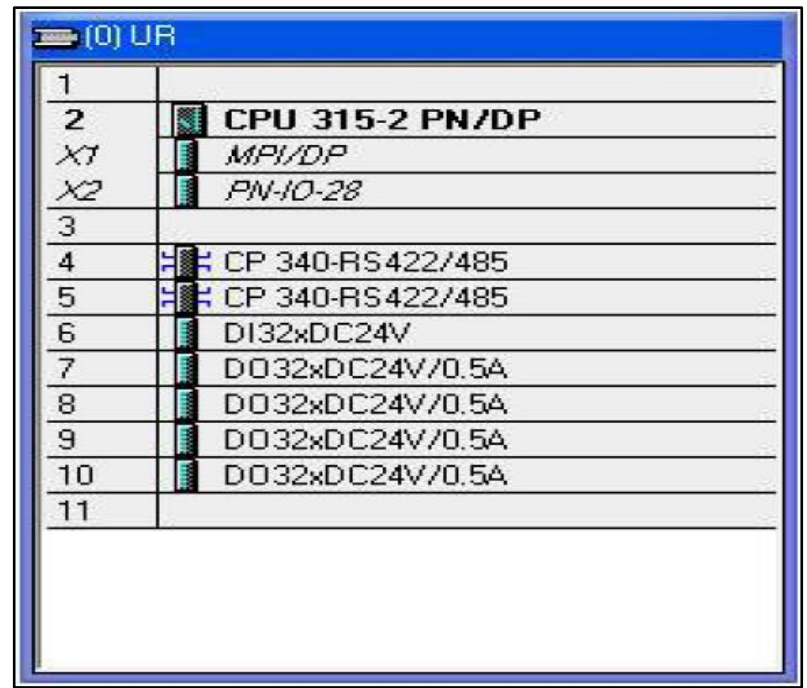

Figura 5: Nueva configuración de Hardware del PLC del RCMS (Vista desde el HW Configuración de STEP7).

Fuente: Autores, (2017).

Cabe destacar que con solo conectar y preparar el SCADA no era necesario llevar a cabo cambios tan sustanciales en este módulo, pero que si nos iba a dotar de una serie de repuestos de gran valor para el país. Permitiendo una rápida respuesta em caso de ser necesario por una avería, evitando la realización de importaciones.

Se modificó todo el programa para este RCMS siendo el único en el país en cada uno de sus aspectos. Por la distancia desde el cuarto de control a las máquinas se modifica también la arquitectura de la red.

\section{III.3 ADECUACIÓN DEL SCADA PARA OPERAR 1 BATERÍA}

Para el sistema supervisorio se utilizó WinCC que constituye el entorno de desarrollo de Siemens en el marco de los SCADA para la visualización y control de los procesos industriales como los de generación de electricidad, refinerías, etc.

Teniendo en cuenta que los elementos de campo utilizados por los fabricantes de los grupos electrógenos con tecnologia Hyundai pertenecen a la firma Siemens, el WinCC V6.2 fue el sistema escogido por los mismos para la supervisión y control de sus subsistemas. Este es un sistema moderno con interfaces cómodas para el usuario, abierto al mundo ofimático y a la producción. Está dotado de funciones probadas y fiables en el ámbito industrial, resulta fácilmente configurable y escalable desde las tareas más sencillas a las más complejas. [1].

El proyecto está estructurado por: las vistas de datos analógicos y eléctricos de los motores, sistemas registradores de los datos eléctricos y analógicos, en los que se incluyen datos del HTU y la Caldera, la navegación de las diferentes pantallas del sistema de combustible, mostrando estados de niveles de los tanques de diesel y fuel en el HTU, estados de las bombas y su operación local y remota.

Ya con los cambios realizados en los módulos de HTU y RCMS fue necesario preparar el sistema SCADA de la planta para su operación. Como principales aspectos se priorizó la eliminación de todas los gráficos y variables que no formaran parte del sistema, así como integrar mímicos para realizar más cómoda y ágil la operación.

Se crearon las nuevas Tags para la integración de las bombas de transferencia de combustible al SCADA que ahora forman parte del autómata del HTU. La configuración de las 
bases de datos para el almacenamiento de las alarmas y eventos de la planta.

\begin{tabular}{|c|c|c|}
\hline JNo1FOTransferPumpRun 1 & Variable binaria & E4.0 \\
\hline IN No1FOTransferPumpalarm1 & Variable binaria & E4.5 \\
\hline NozFotransferPumpRunt & Variable binaria & E4.2 \\
\hline NozFOTransferPumpAlarm1 & Variable binaria & E5.5 \\
\hline NolDoTransferPumpRuni & Variable binaria & $\mathrm{E} 4.3$ \\
\hline No1DOTransferPumpAlarm 1 & Variable binaria & E7.0 \\
\hline NozooTransferPumpRun 1 & Variable binaria & E4.4 \\
\hline No2DOTransferPumpAlarm1 & Variable binaria & E7.7 \\
\hline ZNo1FotransPumpManual1 & Variable binaria & M203.0 \\
\hline NoifoTransPumpManualon & Variable binaria & M203.1 \\
\hline ZNo2FotransPumpManual1 & Variable binaria & M203.2 \\
\hline INozFoTransPumpManualon & Variable binaria & M203.3 \\
\hline Wo1DOTransPumpManual1 & Variable binaria & M203.4 \\
\hline INo1DOTransPumpManualon! & Variable binaria & M203.5 \\
\hline INo2DOTransPumpManual1 & Variable binaria & M203.6 \\
\hline INo20OTransPumpManualon 1 & Variable binaria & M203.7 \\
\hline
\end{tabular}

Figura 6: Nuevas variables incorporadas al HTU para uso en WinCC (Vista desde el Administrador de Variables de WinCC). Fuente: Autores, (2017).

\section{III.4 Descripción de los principales gráficos modificados para el proyecto}

El mímico GPCCtrl.pdl que representa los principales valores eléctricos de cada motor generador, los parâmetros eléctricos (corriente, potencia aparente y energía reactiva generada). Además cuenta con la interfaz de entrada de datos para la operación remota de los generadores.
La pantalla Singleline1.pdl, que representan los esquemas eléctricos de conexión de cada generador con la barra de salida, se encuentran las variables (frecuencia, potencia reactiva, potencia activa, factor de potencia, voltaje, corriente, potencia aparente y potencia aparente a la salida de las baterías y corriente de cada generador) estas son vitales para la operación.

La pantalla EngineAnalog.pdl en la cual se muestran em tiempo real todas las variables analógicas del motor (presión y temperatura de aceite y combustible, temperatura de los gases de escape, presión de aire de arranque entre otros).

Al mostrar los datos de cuatro motores-generadores y tener un solo monitor, se integraron los gráficos de los monolineales con los de control de generadores facilitando el trabajo de sincronización desde el control, al poder visualizar parâmetros eléctricos.

De igual forma en la pantalla EngineAnalog.pdl incorporaron los datos correspondientes al mímico GPCCtrl.pdl es esta la pantalla principal de operación ya que permite al operador visualizar al mismo tiempo todas las variables eléctricas y mecánicas del motor, así como los diferentes estados asociados a las mismas.

Se le incorporó a todos los xxxxxPicWin.pdl del proyecto la opción de autorización de uso, para prevenir con esto la inserción de datos a los autómatas. Esto evita, por ejemplo que si el control del motor-generador no esté pasado hacia el RCMS el operador no pueda accionar los comandos de arranque ó parada de forma remota. Eliminando así una falla importante del proceso.

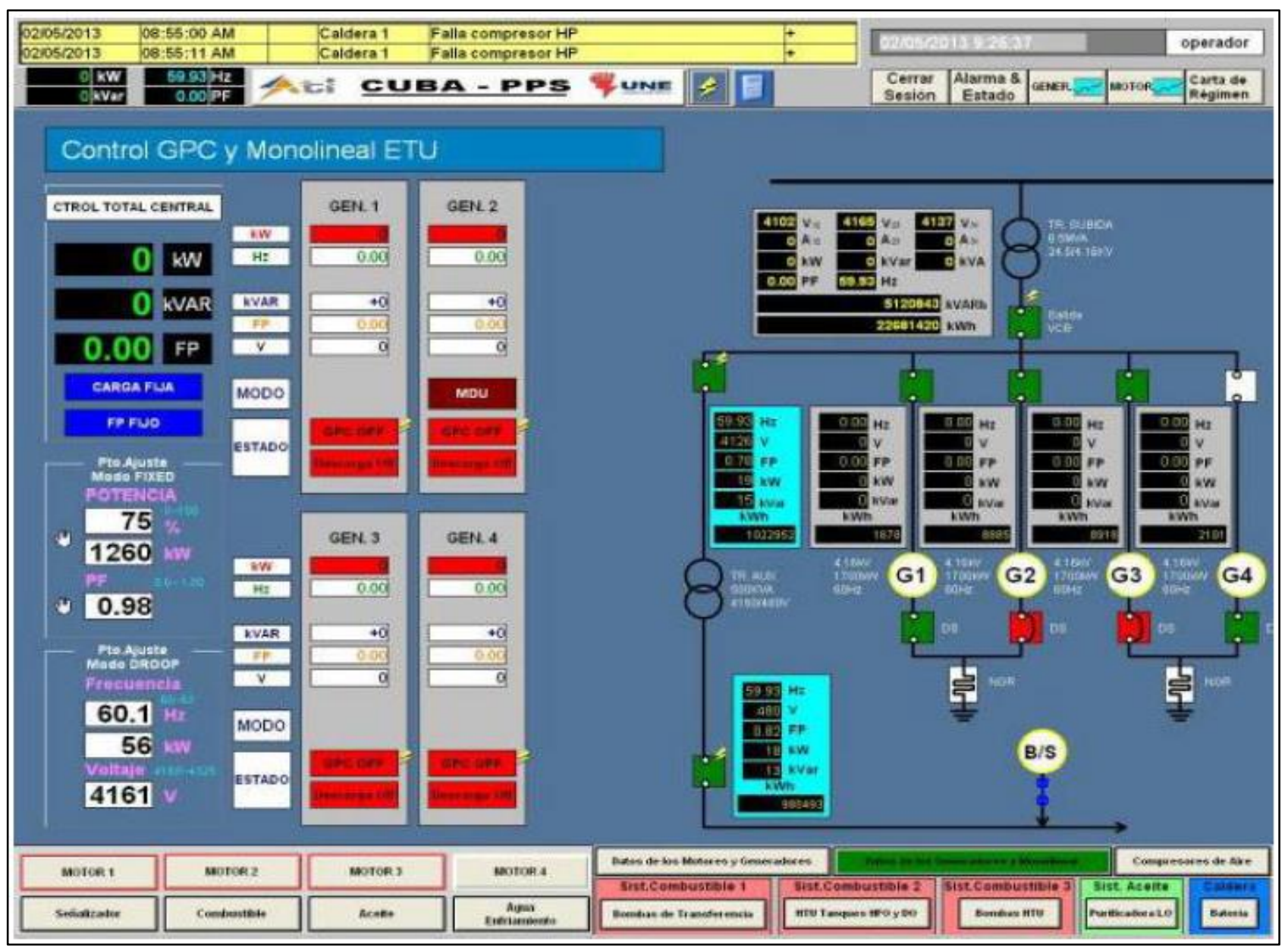

Figura 7: Mímico de Control de los Generadores y Monolineal.

Fuente: Autores, (2017). 


\section{PROGRAMACIÓN DE LOS AUTÓMATAS Y CONFIGURACIÓN DE LA COMUNICACIÓN}

Los sistemas de Hyundai cuentan con autómatas Siemens S7-300 para sus configuraciones de 1,7 MW y 2,5 MW que son las que disponemos en Cuba.

La transferencia de datos se lleva a cabo con Ethernet Industrial. Mediante bloques de función de Siemens FB12 "BSEND" para envió y FB13 "BRCV" para recepción.

El FB 12 (BSEND) emite datos a un FB remoto del tipo "BRCV". En esa transferencia de datos puede ser transportada una mayor cantidad de datos entre los interlocutores de lo que es posible entre todos los demás FB para los enlaces S7 configurados, o sea, hasta 32768 bytes en S7-300 [4].

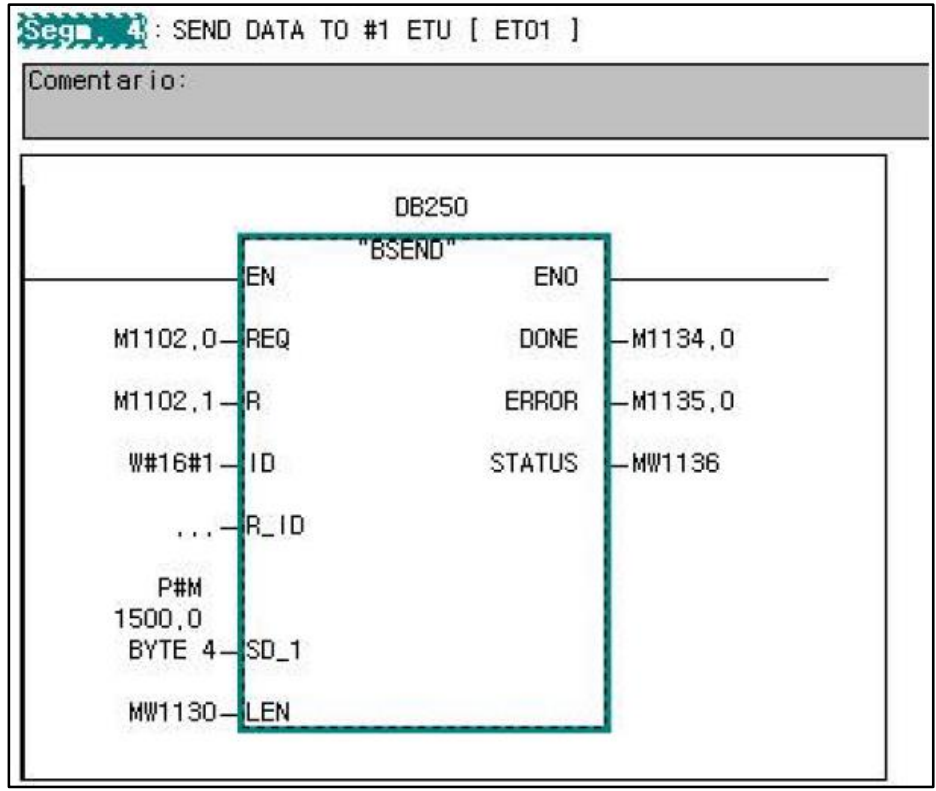

Figura 8: Ejemplo de envió desde PLC del RCMS al PLC de ETU.

Fuente: Autores, (2017).
El FB 13 (BRCV) recibe datos de un FB remoto associado del tipo "BSEND". Después de cada segmento de datos recibido se envía un acuse de recibo al FB asociado [4].

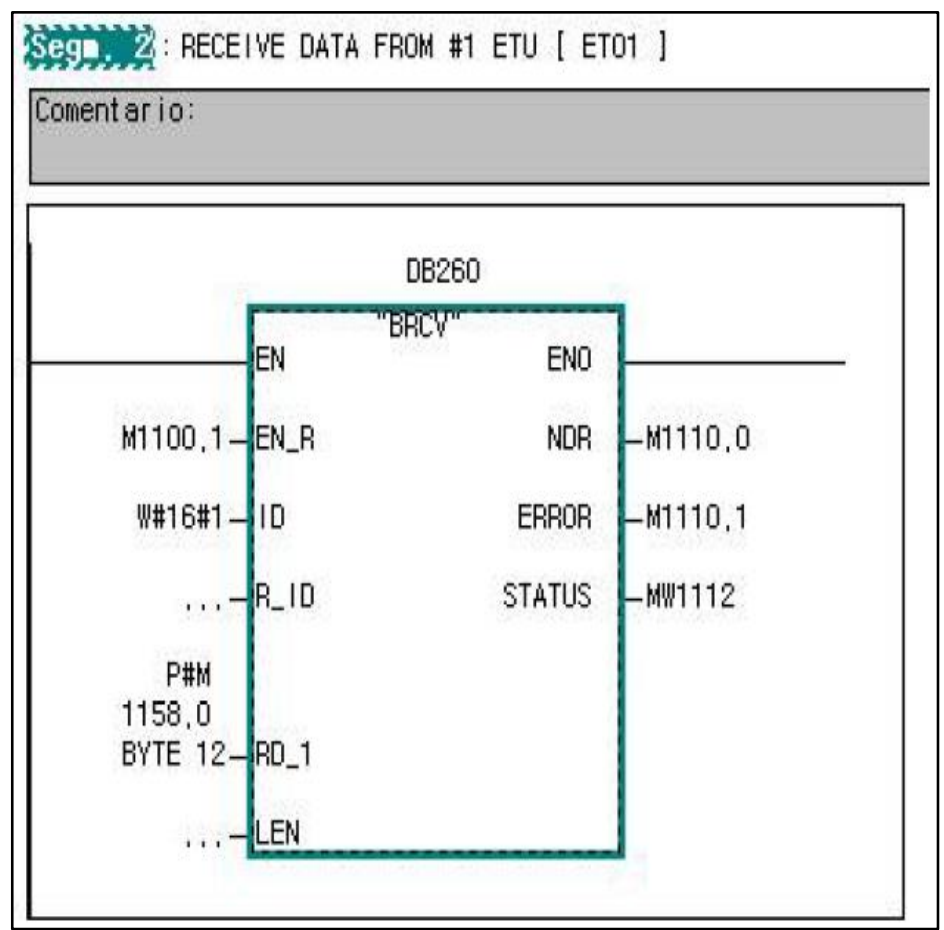

Figura 9: Ejemplo de recepción del PLC del RCMS desde el PLC de ETU.

Fuente: Autores, (2017).

Se enlazan por cada cuatro unidades motor - generador cada una de sus CPU a un switch concentrador, la CPU de la unidad unidades que son comunes a la central.

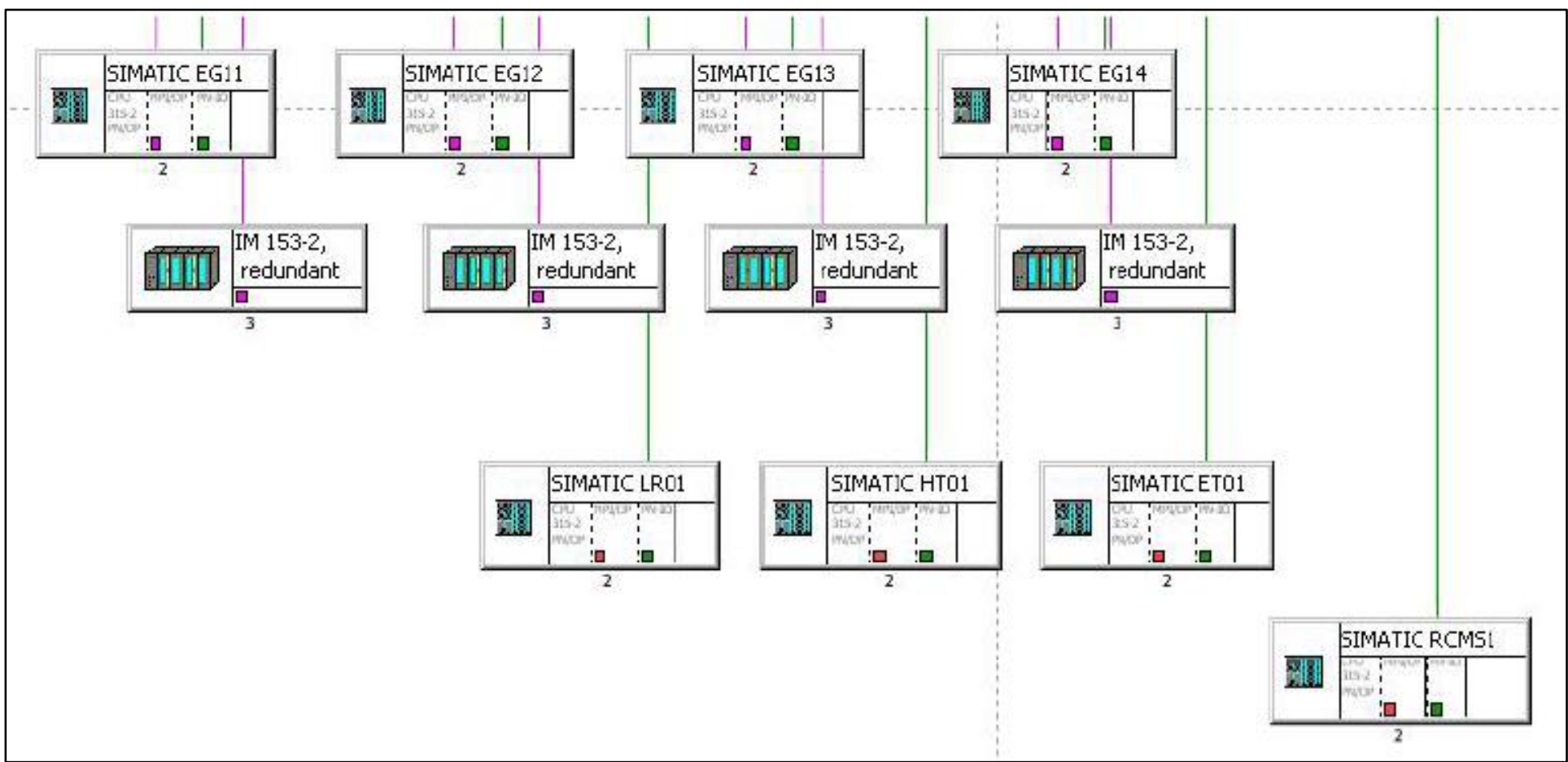

Figura 10: Arquitectura de la comunicación de Ethernet Industrial (Vista desde el NetPro de STEP7).

Fuente: Autores, (2017). 
Este Switch se enlaza con otro que concentra en la estación de monitoreo las salidas homólogas de las restantes unidades motor - generador y de la CPU de la propia estación de monitoreo, de donde se enlazan las PC industriales para de esta forma manipular las variables que llegan al SCADA.

\begin{tabular}{||l|lll|}
\hline ID local & ID del interloc & Interlocutor & Tipo \\
\hline 1 & 4 & SIMATIC EG11/CPU 315-2 PNIDP & Enlace S7 \\
\hline 2 & 4 & SIMATIC EG12 / CPU 315-2 PNIDP & Enlace S7 \\
3 & 4 & SIMATIC EG13/CPU 315-2 PNIOP & Enlace S7 \\
4 & 4 & SIMATIC EG14/CPU 315-2 PNIDP & Enlace S7 \\
5 & 5 & SIMATIC HT01/CPU 315-2 PNIDP & Enlace S7 \\
6 & 5 & SIMATIC LR01/CPU 315-2 PNIDP & Enlace S7 \\
7 & 1 & SIMATIC RCMS1 / CPU 315-2 PNIDP & Enlace S7 \\
\hline
\end{tabular}

Figura 11: Enlaces de la comunicación de Ethernet Industrial del PLC de ETU (Vista desde el NetPro de STEP7).

Fuente: Autores, (2017).

Con Modbus 485 se enlazan cada uno de los GPC de cada cuatro unidades motor - generador el cual tiene la función del reparto de carga de estas así como las protecciones eléctricas. Por Modbus 485 de igual forma se enlazan cada uno de los metros de energías. Ambos enlaces se conectan a través de um módulo de expansión (CP340) de la CPU de la estación de monitoreo. La información es manipulada y enviada por Industrial Ethernet al Switch donde las PC leen estos datos. Com Profibus solo se enlazan las CPU de cada unidad motor - generador con sus módulos de entrada y salida a través de la tarjeta de interface remota [1].

\section{CONCLUSIONES}

Este proyecto permitió profundizar en los conocimientos sobre la tecnología Hyundai, la programación de los PLC y la implementación de los SCADA, lo que posibilitó la modificación de un proyecto para realizar un uso más eficiente de este.

El acoplamiento de la batería Hyundai incrementó la potencia instalada al sistema aislado de Cayo Santa María. Las modificaciones al sistema SCADA y la programación de los PLC, contribuyó de manera general a la sustitución de importaciones

\section{RECOMENDACIONES}

Estudiar la implementación del control de la carga de los generadores de forma independiente para explotar con mayor eficiencia la capacidad del sistema SCADA.

\section{REFERENCIAS}

[1] Configuración CP 340 RS232. [en línea]. http://plc-hmiscadas.com/128.php (Consulta 20 enero, 2013).

[2] Curso de WinCC [en línea]. http://www.infoplc.net/Descargas/Descargas_Siemens/Des_Siem ensFile

s /infoPLC_net_GettingStartedWinCC.html. (Consulta 20 junio, 2010).

[3] Esquema de contactos (KOP) para S7-300 y $\mathbf{S 7 - 4 0 0 .}$ Manual de referencia. 6ES7810-4CA07-8DW1. Edición 01/2004

[4] Formación en Automatización y Sistemas SIMATIC S7. Ethernet S7.(2012)

[5] Formación en Automatización y Sistemas SIMATIC S7. Mantenimiento.(2012)
[6] Formación en Automatización y Sistemas SIMATIC S7. Programación 1 Curso S7 Nivel 1. (2012)

[7] Formación en Automatización y Sistemas SIMATIC S7. Programación2. Curso S7 Nivel 2. (2012)

[8] Instruction book Volume V. Engine type 9H21/32. Hyundai Heavy Industries Co., Ltd. HIMSEN, HYUNDAI. [CD-ROM]. (2012)-. KOREA. Publicación seriada.

[9] Instruction book Volume VIII. Engine type 9H21/32. Hyundai Heavy Industries Co., Ltd. HIMSEN, HYUNDAI. [CD-ROM]. (2012)-. KOREA. Publicación seriada.

[10] Instruction book Volume IX. Engine type 9H21/32. Hyundai Heavy Industries Co., Ltd. HIMSEN, HYUNDAI. [CD-ROM]. (2012)-. KOREA. Publicación seriada.

[11] Instruction book Volume XII. Engine type 9H21/32. Hyundai Heavy Industries Co., Ltd. HIMSEN, HYUNDAI. [CD-ROM]. (2012)-.KOREA. Publicación seriada.

[12] O. Deiner Más. "Desarrollo y ampliación de funcionalidades del proyecto SCADA en grupos electrógenos de fuel oíl con tecnología Hyundai". Artículo revista GIGA, Número 1/2011. ISSN: 1028-270x.

[13] Operación y Supervisión con WinCC. Curso del sistema ST-BWINCCS. 2012.

[14] WinCC [CD-ROM].Ver. 6.2 sp3 [ASIA]: HYUNDAI, 2006. Manual de Ayuda. [Consulta abril, 2012]. 